\title{
Morphological and Mechanical Biomimetic Bone Structures
}

\author{
R. Parwani, ${ }^{\dagger}$ M. Curto, ${ }^{\dagger}$ A. P. Kao, ${ }^{\dagger}$ P. J. Rowley, ${ }^{\dagger}$ M. Pani, ${ }^{\dagger}$ G. Tozzi, ${ }^{\dagger}$ and A. H. Barber* ${ }^{\dagger} \odot$ \\ ${ }^{\dagger}$ School of Engineering, Anglesea Building, Anglesea Road, University of Portsmouth, Portsmouth PO1 3DJ, United Kingdom \\ ${ }^{\ddagger}$ School of Earth and Environmental Sciences, Burnaby Building, Burnaby Road, University of Portsmouth, Portsmouth PO1 3QL, \\ United Kingdom
}

\begin{abstract}
Cortical bone is an example of a mineralized tissue containing a compositional distribution of hard and soft phases in 3-dimensional space for mechanical function. X-ray computed tomography (XCT) is able to describe this compositional and morphological complexity but methods to provide a physical output with comparable mechanical function is lacking. A workflow is presented here to establish a method of using high contrast XCT to establish a virtual model of cortical bone that is manufactured using a multiple material capable 3D printer. Resultant 3D printed structures were produced based on more and less remodelled bone designs exhibiting a range of secondary osteon density. Variation in resultant mechanical properties of the $3 \mathrm{D}$ printed composite structures for each bone design was achieved using a combination of material components and reasonable prediction of elastic modulus provided using a Hashin-Shtrikman approach. The ability to $3 \mathrm{D}$ print composite structures using high contrast XCT to distinguish between compositional phases in a biological structure promises improved anatomical models as well as nextgeneration mechano-mimetic implants.

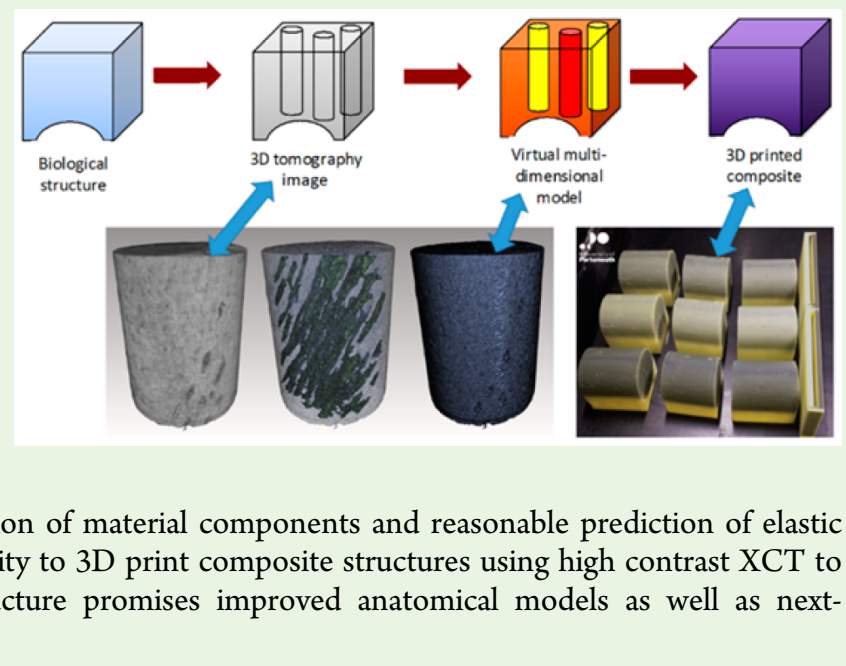

KEYWORDS: bone, mechanics, 3D printing, additive manufacturing, X-ray tomography, composites

\section{INTRODUCTION}

Biological processes are adept at producing complex structures optimized for a range of mechanical functions, while maintaining biological function. Such structural complexity requires both morphological and compositional control often lacking in synthetic routes. Bone is a prevalent example of a mineralized tissue demonstrating considerable mechanical performance, including resistance to compression and relatively high toughness, ${ }^{1}$ by means of an optimized combination of hard mineral apatite and a range of softer materials mostly consisting of collagen. ${ }^{2-5}$ Bone is commonly classified into a number of hierarchical levels from the whole bone down to nanoscale components. ${ }^{6}$ A number of disease states and conditions exist that compromise the mechanical integrity of bone, mainly including osteoporosis and osteoarthritis. ${ }^{7,8}$ Considerable improvement in healthcare therefore requires effective replacement of bone material that is able to provide suitable mechanical function.

The replacement of bone broadly follows two pathways of either employing biomaterials for bone tissue engineering to allow bone regeneration ${ }^{8}$ or using engineering structures to replace significant volumes of the whole bone via traditional total hip or knee replacements. ${ }^{9}$ The former design of biomaterials has become a sophisticated research field that employs a range of solutions that are mostly suited for small defects, whereas larger structures are perhaps less developed. ${ }^{10}$ Specifically, prosthetics are typically employed to interface with bone material but lack the morphological and compositional complexity comparable to that of the host material. This lack in complexity often results in failure of the implant, mainly due to aseptic loosening. ${ }^{11}$

Manufacturing processes able to provide complexity in order to satisfy mechanical function comparable to bone are limited. Additive layer manufacturing, commonly referred to as threedimensional (3D) printing, shows significant potential in producing the complexity required for mimicking bone, or indeed any biological structure. Extensive efforts have been made in applying $3 \mathrm{D}$ printing to a range of biological structure related areas. Healthcare, particularly in surgery, is an area of significant growth for structures produced from $3 \mathrm{D}$ printing. ${ }^{12}$ Highlighted use of $3 \mathrm{D}$ printing includes the manufacture of anatomic models ${ }^{13}$ as well as surgical guides and templates, ${ }^{14}$ implants, ${ }^{15}$ and molds predominantly for maxillofacial and orthopedic operations. ${ }^{16}$ Interestingly, a recent review indicates the activity in $3 \mathrm{D}$ printing of anatomic models was over seven times larger than for implant studies ${ }^{17}$ and perhaps reflects the demands in controlling the biological and mechanical function of an implant compared to a model. The aim of manufacturing a bone replica mimicking the host tissue using $3 \mathrm{D}$ printing

Special Issue: Multiscale Biological Materials and Systems: Integration of Experiment, Modeling, and Theory

Received: October 21, 2016

Accepted: December 19, 2016

Published: December 19, 2016 

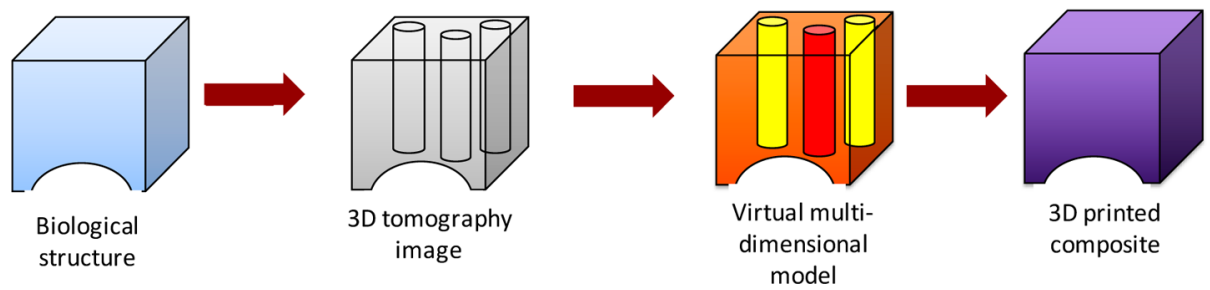

Figure 1. Workflow employed to 3D print a bone structure exhibiting morphological and mechanical fidelity with the host biological structure. XCT is first applied to provide a 3D tomography image of the cortical bone structure. The 3D image data contains both compositional and morphological information that is translated to a virtual multidimensional model incorporating morphological information as well as assigning mechanical properties of the primary and secondary osteonal regions. A physical output of this virtual model is provided by the 3D printer.

therefore remains a significant goal both for providing increasingly effective implants as well as more accurate anatomic models.

Previous works have highlighted the use of $3 \mathrm{D}$ printing in presenting engineered structures based on biological composite topologies including a rotated bone-like geometry. ${ }^{18} 3 \mathrm{D}$ printing critically requires a design input that is realized in a physical model output. Such an input ranges from computer aided design $(\mathrm{CAD})^{13}$ to more sophisticated use of X-ray computed tomography (XCT) imaging. ${ }^{19}$ The latter approach is powerful as the three-dimensional complexity of a biological structure is captured depending on the resolution and field of view. A less developed aspect of XCT scanning resides in capturing both geometric information as well as compositional information based on the attenuation between the probing incident X-ray and the materials organized within the biological structure. The ability to obtain a digital model of bone that maintains high fidelity with the host tissue using XCT is persuasive. Although $3 \mathrm{D}$ printing shape information from bone has been achieved, ${ }^{20}$ the use of multiple material 3D printing of biological structures is lacking. Prevalent examples of $3 \mathrm{D}$ printed multimaterial structures inspired by nature exist for the nacreous layer of sea shells that consist of a high volume fraction of hard mineral plates, within softer material referred to as a "bricks and mortar" organization. ${ }^{21}$ The challenge of accurately manufacturing volume fractions above $90 \%$ of hard material within a softer matrix material still remains, but works have indicated a broader approach that allows a mimetic hardsoft material composite with functionality that tends toward that of the host biological structure. ${ }^{18}$ However, the integration of an efficient workflow that allows information translation from XCT to a virtual model that gives a 3D printed physical output with mechanical fidelity from shape and composition is required. This work presents such an integrated approach demonstrated for compact bone structures. Compact bone is a demanding biological structure for XCT as the solid volume fraction is high, with few voids that provide high contrast at interfaces with the solid mineralized material. Compact bone that is remodelled also gives opportunities to examine regions of compositional variations between secondary osteons compared to the primary osteonal bulk. The potential for 3D printing structures that retain the characteristics of the host biological tissue additionally require selection of appropriate materials with a distribution of mechanical properties that enable suitable function. Although the establishment of a workflow approach from imaging through to manufacturing is critical, the development of future materials is expected to give increased fidelity. A 3D printed structure directly using biological design must finally provide mechanical function comparable to the host to achieve a "mechano-mimetic" goal.
The main aim of this study is to establish a workflow able to provide a physical 3D printed output of a bone structure using $\mathrm{XCT}$ approaches. The resultant structural output is primarily exploiting the power of $3 \mathrm{D}$ printing in giving organizational complexity of materials, but utilizes commercially available materials. Materials with a range of mechanical properties will explore the ability to tune composition combined with structural fidelity to approach a more mechano-mimetic 3D printed bonelike structure.

\section{EXPERIMENTAL SECTION}

Cortical bone samples were harvested from mature bovines that were bred and slaughtered for alimentary purposes. Typical age of sacrifice in dairy cows ranges 36-48 months and this is considered as the "biological" age of the samples used in the current study. Samples from the mid-diaphysis of bovine bone femura were cored by removing cylinders of approximate diameters and lengths of $4 \mathrm{~mm} \times 5 \mathrm{~mm}$ respectively from the host. The long axis of the cored cylinder was parallel to the long axis of the bovine bone femur. Cored samples were extracted from an extensively remodelled bovine bone region showing a significant number of secondary osteons and a less remodelled bovine bone region limiting the number of secondary osteons. Cored bone samples were wrapped in saline soaked gauze and frozen prior to imaging.

The approach taken here is to image the samples using XCT to give morphological information and identify the compositional variations of primary and secondary bone. Primary bone is produced rapidly in bovine structures but is remodelled into more ordered secondary osteonal regions. While the composition of bone is predominately hard mineral phase and softer collagen, regions of disordered bone have been shown to have relatively lower stiffness ${ }^{5}$ whereas more ordered bone exhibits increased stiffness. ${ }^{22}$ A workflow is therefore established as indicated in Figure 1 to image cortical bone samples with regions of compositional variation and then develop a virtual model of the bone, including morphological and mechanical information, which is translated to a $3 \mathrm{D}$ printed composite structure of multiple materials exhibiting an organizational fidelity with the host tissue.

Imaging of bone was carried out using an $\mathrm{X}$-ray microscope (Versa 520, Carl Zeiss Ltd., USA) operating with a $70 \mathrm{kV} / 6 \mathrm{~W}$ X-ray tube energy. A $5.3 \mu \mathrm{m}$ isotropic voxel size was achieved from imaging samples using a total of 3201 projections across 360 deg of sample rotation. Each projection was collected using a $6 \mathrm{~s}$ exposure time. The core samples were immersed in saline during the tomography to prevent desiccation. The $2 \mathrm{D}$ X-ray projections from XCT were reconstructed to a $3 \mathrm{D}$ volume using a filtered back projection algorithm implemented in the manufacturers software. A standard Shepp-Logan filter, Gaussian filter ( 0.5 strength) and beam hardening correction (strength of 0.05 for the more remodelled bone and 0.044 for the less remodelled bone) was applied to the projections. Approximately 50 slices from the top and bottom regions of the XCT data sets were disregarded due to artifacts. The resulting 3D data set was segmented into primary and secondary bone regions by machine learning based segmentation (Weka 3.9.0, ImageJ, U. S. 

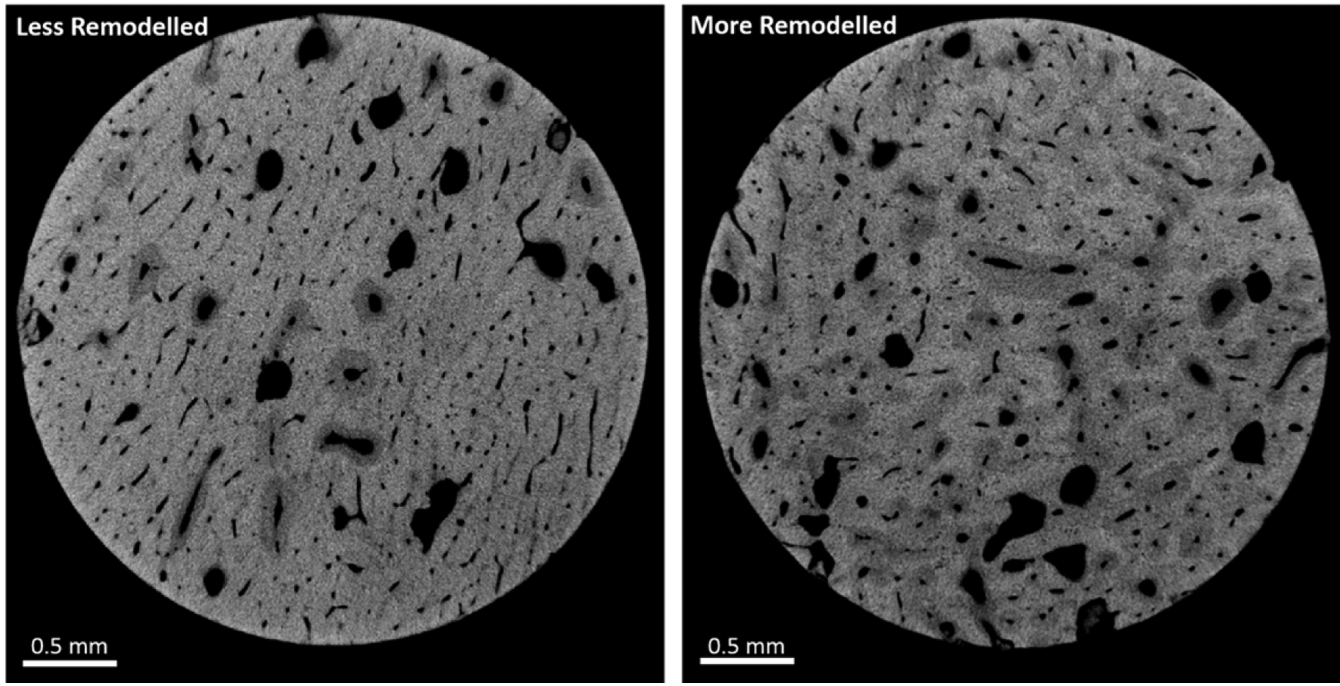

Figure 2. 2D virtual slices of the 3D tomography data generated from the XCT highlighting less remodelled (left) and more remodelled (right) cortical bone structure. Extensive secondary osteon regions are shown around the pores of the more remodelled bone, whereas more limited numbers of secondary osteon regions are seen in the less remodelled cortical bone.
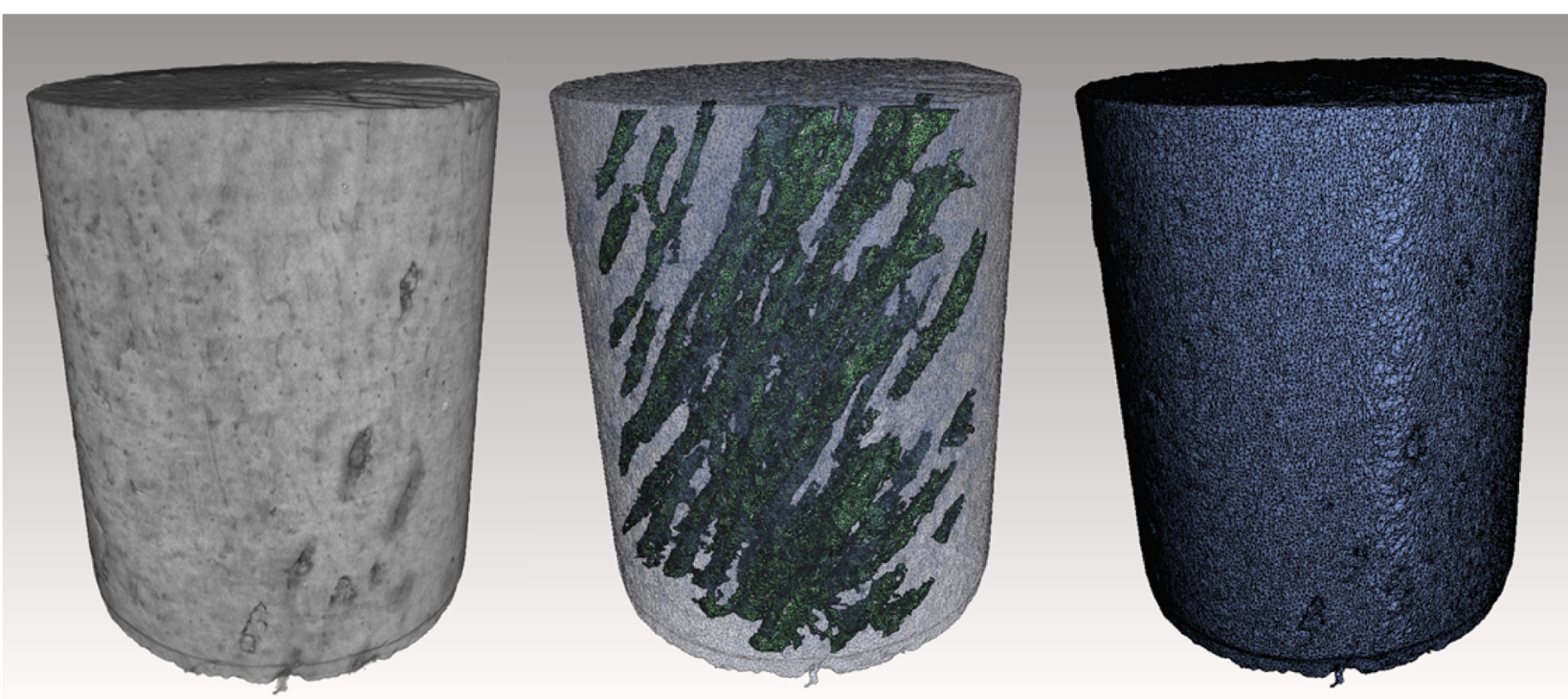

Figure 3. Virtual model development first used the X-ray tomograms (left) and resultant segmented data (middle) to distinguish the primary bone from the tubular features of the secondary osteons. Meshing (right) gave a complete model that was suitable for a physical output from $3 \mathrm{D}$ printing that retains compositional and morphological information.

National Institutes of Health, Bethesda, Maryland, USA) to distinguish between the texture of primary and secondary osteons. This segmentation gave a $3 \mathrm{D}$ analogue of the data sets using image analysis software (Avizo 8, Fra.). An isosurface was extracted from the 3D analogue and triangulated as a mesh of polygons and volume meshes using meshing software (MeshLab v1.3.3., Ita.). Each mesh was decimated in terms of triangle number with a multistep procedure defined by a step number equal to three. A quadric edge collapse decimation algorithm with a quality threshold parameter of 0.5 and a boundary preservation weight of 5 was used to decimate the mesh to reduce data size. Meshes were imported into CAD software (Rhinoceros 5.0, Robert McNeel and Associates, USA) and scaled by $\times 10$ to increase feature density within the 3D printed structure. Validation of the closed surface of the meshing and removal of hole artifacts was carried out using software (NetFabb, Autodesk, UK). Finally, 3D printed samples were outputted from the CAD to a physical composite model using an inkjet based 3D printer (ProJet 5500X, 3D Systems, USA) that allowed the additive deposition of multiple materials. The hardest material was used for the secondary bone regions (Visijet CR-WT, 3D Systems, USA) and a series of increasingly softer matrix materials (VisiJet RWT-EBK 100, VisiJet RWT-EBK 250 and VisiJet RWT-EBK 500, 3D Systems, USA) defined as hard, medium and soft respectively were used as the primary bone material. The materials were chosen from the range available commercially for use in the $3 \mathrm{D}$ printer. The approximate ratios of the hardest to the increasingly softer materials using the manufacturers elastic modulus specifications are $2.7,11$, and 40 , respectively. The ratio of elastic modulus for the secondary osteonal material compared to primary osteon is approximately 10 , taken from literature, ${ }^{5}$ indicating a ratio of $3 \mathrm{D}$ printed materials comparable to those found in bone despite the absolute values being lower. These $3 \mathrm{D}$ printed base materials are noted as USP Class VI certified for healthcare applications. The printer was operated in XHD mode with a $13 \mu \mathrm{m}$ spatial resolution is the $z$-axis and $34 \mu \mathrm{m}$ spatial resolution in the $x$ and $y$-axes of the build plate plane. The long axis of the cortical bone structure was aligned along the $x$-axis. XCT validation of the $3 \mathrm{D}$ 


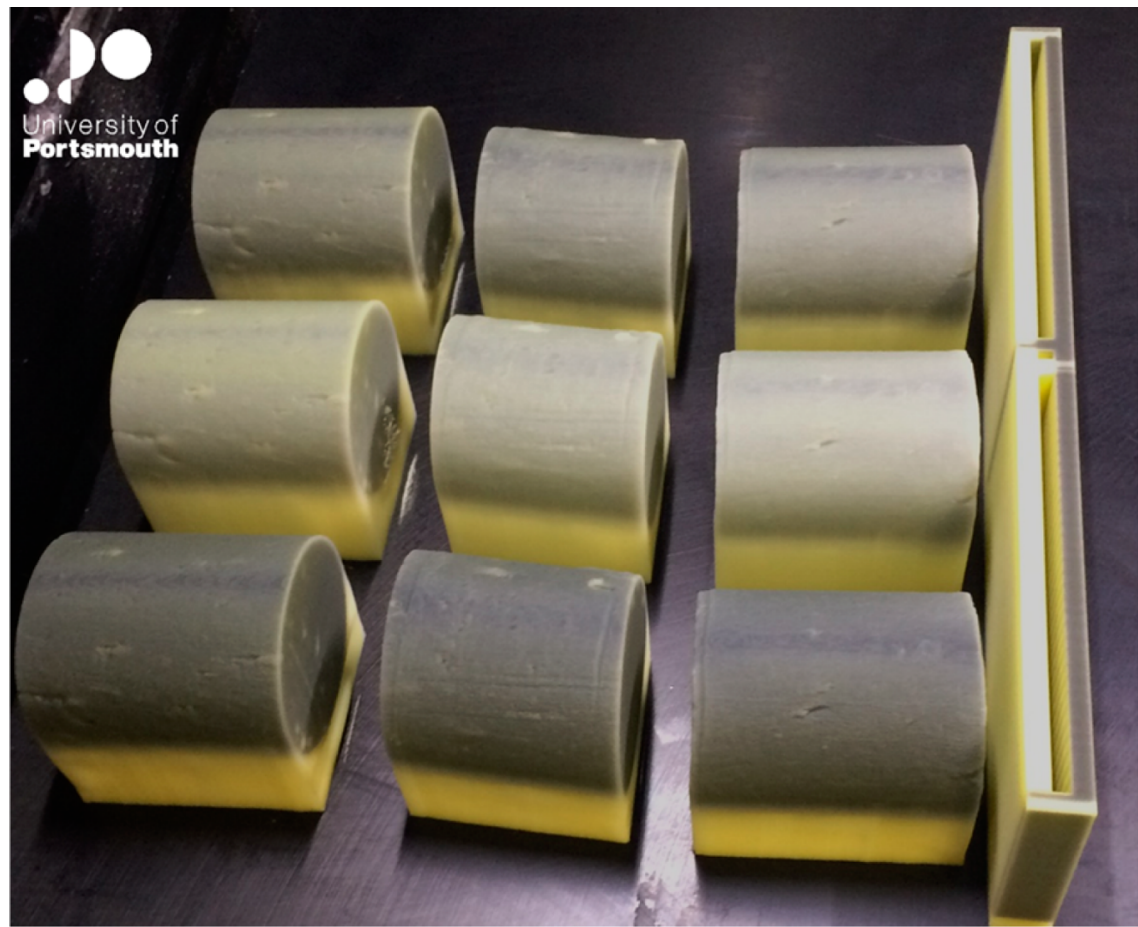

Figure 4. Optical image showing the 3D printing bovine bone structures from XCT data. The printed material is effectively the darker coloration whereas the wax support is the lighter region underneath the sample. Note the long axis of the bone is left to right in the image and parallel to the build plate of the $3 \mathrm{D}$ printer.

printed samples was attempted but was impossible to distinguish between the different material compositions due to similarity of X-ray attenuation across all the base materials.

Mechanical properties of the cortical bone samples and 3D printed mimics were evaluated using acoustic measurements. The propagation of ultrasonic waves is an established method of measuring the elastic properties of bone as well as $3 \mathrm{D}$ printed trabecular bone phantoms as demonstrated recently. ${ }^{20}$ Samples of bone and 3D printed structures were fixed between a transmitting and receiving transducer setup (Olympus V103/V153, UK). The transducers were clamped using coupling media (ShearGel, Magnaflux, USA) to the opposite ends of the samples using an approximate force of $10 \mathrm{~N} . \mathrm{cm}^{2}$ so that the long axis of the sample traversed between the transducers. A $1 \mathrm{MHz}$ sinepulse was generated, with a repetition frequency between 10 and $1000 \mathrm{~Hz}$, at the transmitted end of the sample so that the ultrasonic pulse was detected at the receiver using an oscilloscope. The fast first arrival ultrasonic wave velocity, define as the primary p-wave velocity $V_{\mathrm{p}}$, and secondary s-wave velocity $V_{\mathrm{s}}$ were calculated using

$$
\begin{aligned}
& V_{\mathrm{p}}=L t_{\mathrm{p}}^{-1} \\
& V_{\mathrm{s}}=L t_{\mathrm{s}}^{-1}
\end{aligned}
$$

Where $t_{\mathrm{p}}$ and $t_{\mathrm{s}}$ are the $\mathrm{p}$ - and s-wave arrival times, and $L$ is the sample length. The apparent elastic modulus $E$ of the cortical bone is calculated from ${ }^{23}$

$$
E=2 \rho V_{\mathrm{s}}^{2}\left[1+\left(\frac{V_{\mathrm{p}}^{2}-2 V_{\mathrm{s}}^{2}}{2\left(V_{\mathrm{p}}^{2}-V_{\mathrm{s}}^{2}\right)}\right)\right]
$$

Where $\rho$ is the sample density given from volumetric methods.

\section{RESULTS}

Complete volumes of the bovine bone were successfully imaged using XCT for both the less remodelled and more remodelled cortical bone samples. Figure 2 shows plane sections orthogonal to the long axis of the bone and indicates the prevalence of the tubular secondary osteon regions in the more remodelled bone and an absence of secondary osteon regions in the less remodelled bone. The $3 \mathrm{D}$ tomography data sets for less and more remodelled bone samples were used to provide a virtual model of the bone following a series of steps as shown in Figure 3. The 3D data was segmented to highlight the secondary osteons and then finally meshed with a range of triangular features from approximately 1.5 million for the less remodelled bone to 3 million for the more remodelled bone. The increased digital weight for the more remodelled bone compared to the less remodelled bone was due to the increased number of secondary osteon features in the mesh. The 3D printed physical output from the virtual model is shown in Figure 4 for a number of samples. The 3D printing provides a low-density wax material support that is observed as the lighter coloration under the darker structural material.

Mechanical evaluations of the base materials used to construct the $3 \mathrm{D}$ printed structures of bovine-like bone are shown in Table 1. Minimal variations of both $\mathrm{p}$ - and s- wave velocities between the hardest and hard materials resulted in similar elastic modulus values of 3.95 and $3.85 \mathrm{GPa}$, respectively. A reduction of $16 \%$ in elastic modulus is observed

Table 1. List of the P-And S- Wave Velocities and Corresponding Calculated Elastic Modulus Values for a Range of the 3D Printed Base Materials Used

$\begin{array}{lcccc} & \begin{array}{c}\text { p-wave velocity } \\ \left(\mathrm{m} \mathrm{s}^{-1}\right)\end{array} & \begin{array}{c}\text { s-wave velocity } \\ \left(\mathrm{m} \mathrm{s}^{-1}\right)\end{array} & \begin{array}{c}\text { density } \\ \left(\mathrm{g} \mathrm{cm}^{-3}\right)\end{array} & \begin{array}{c}\text { elastic } \\ \text { modulus } \\ (\mathrm{GPa})\end{array} \\ \text { hardest } & 2401 & 1100 & 1.19 & 3.95 \\ \text { hard } & 2367 & 1073 & 1.22 & 3.85 \\ \text { medium } & 2269 & 997 & 1.18 & 3.24 \\ \text { soft } & 2155 & 921 & 1.15 & 2.71\end{array}$


between the hard and medium materials with a further $17 \%$ reduction in elastic modulus for the soft material. Elastic modulus measurements for the 3D printed structures and the corresponding less and more remodelled bone samples are shown in Table 2. The bone samples exhibit noticeably higher

Table 2. List of the P-And S-Wave Velocities and Corresponding Calculated Elastic Modulus Values for the Less and More Remodelled Cortical Bone Samples, and the Corresponding 3D Printed Composite Structures with a Range of Matrix Materials

\begin{tabular}{lcccc} 
& $\begin{array}{c}\text { p-wave } \\
\text { velocity } \\
\left(\mathrm{m} \mathrm{s}^{-1}\right)\end{array}$ & $\begin{array}{c}\text { s-wave velocity } \\
\left(\mathrm{m} \mathrm{s}^{-1}\right)\end{array}$ & $\begin{array}{c}\text { density } \\
\left(\mathrm{g} \mathrm{cm}^{-3}\right)\end{array}$ & $\begin{array}{c}\text { elastic } \\
\text { modulus } \\
(\mathrm{GPa})\end{array}$ \\
$\begin{array}{c}\text { more } \\
\text { remodelled }\end{array}$ & 4591 & 2104 & 2.65 & 32.07 \\
$\begin{array}{c}\text { hard matrix } \\
\text { medium }\end{array}$ & 2409 & 1101 & 1.21 & 4.00 \\
matrix & 2331 & 1070 & 1.19 & 3.72 \\
$\begin{array}{l}\text { soft matrix } \\
\text { less } \\
\text { remodelled }\end{array}$ & 2290 & 988 & 1.18 & 3.18 \\
$\begin{array}{l}\text { hard matrix } \\
\text { medium } \\
\text { matrix }\end{array}$ & 2260 & 2415 & 2.04 & 31.74 \\
soft matrix & 2194 & 1027 & 1.20 & 3.45 \\
\hline
\end{tabular}

elastic moduli than the $3 \mathrm{D}$ printed structures and is expected to be due to the high elastic modulus, reported as $129 \mathrm{GPa}^{4}$, of the mineral phase in bone. Interestingly, the less and more remodelled cortical bone samples have similar elastic moduli. The variation of the secondary osteon composition of the less and more remodelled bone is clearly shown in Figure 2, with analysis of the 3D tomographs indicating volume fractions of 4 and 55\%, respectively, for less and more remodelled bone. However, the volume fraction porosity of the more remodelled bone is slightly higher at $8 \%$ than the $7 \%$ for less remodelled bone. The porosity of the less and more remodelled bone samples as well as their corresponding $3 \mathrm{D}$ printed designs was taken from the XCT imaging data sets and calculated using volume fraction analysis (Visual SI Advanced, ORS, Can.). The more and less remodelled porosity volume fraction was found to be 7.12 and $6.63 \%$ respectively. The corresponding average volume fraction porosity from the $3 \mathrm{D}$ printed samples for the more and less remodelled designs was 5.47 and $4.24 \%$, respectively. The bone samples show a slight increase in porosity from the less to more remodelled bone. The 3D printed samples show the same trend of increasing porosity moving from the less remodelled to the more remodelled bone design. The lower porosity for the $3 \mathrm{D}$ printed samples compared to the bone is expected to be due to the meshing process removing small pores that are below the mesh size prior to the $3 \mathrm{D}$ printing. We also note that the voxel size of over 5 $\mu \mathrm{m}$ may also ignore submicrometer porosity in bone linked to the larger scale porosity. The increase in the stiffer secondary osteon phase of more remodelled bone is thus potentially offset by the enhanced porosity relative to the less remodelled bone. An attempt to understand the variation in the mechanical properties of the $3 \mathrm{D}$ printed structures was attempted by plotting the ratio of hard osteonal-like regions to softer matrix against the measured elastic modulus in Figure 5. A linear trend of increasing measured elastic modulus with decreasing ratio was observed for both the less and more remodelled designs. This trend is reasonable as the replacement of a soft matrix with

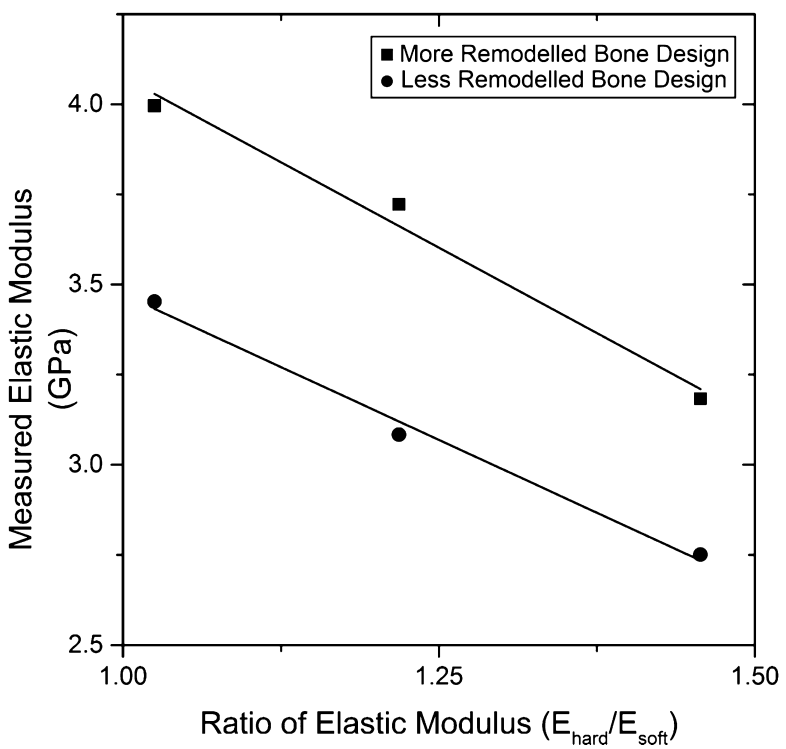

Figure 5. Plot of the variation in the measured elastic modulus of $3 \mathrm{D}$ printed structures based on less and more remodelled cortical bone design with the ratio of elastic moduli of the hard to soft materials used in these structures.

materials of higher elastic modulus occurs when moving from the soft to the hard matrix material. The higher volume fraction of osteonal-like material for the more remodelled bone is reflected in the higher elastic modulus of the structures using the corresponding bone design relative to the less remodelled bone design.

\section{DISCUSSION}

A workflow providing a manufactured realization of the XCT imaging data has enabled composition and morphology to be captured using multimaterial 3D printing. The less and more remodelled bone designs provided morphological information with the selection of a range of base materials providing compositional variation in a $3 \mathrm{D}$ printed bone-like structure. While the measured elastic modulus of 3D printed structures are almost ten times smaller than that of the native bone, the potential to increase the elastic modulus of the overall structure is achievable provided higher elastic modulus base materials are used.

An analytical model able to describe the link between the composition of the 3D printed structures and measured elastic modulus, for each cortical bone design, is explored here in order to understand the potential for tuning mechanical properties toward a more mechano-mimetic structure. A Hashin-Shtrikman description of a composite system of softer matrix and harder phase of homogeneous, isotropic and arbitrary geometry was considered as appropriate. ${ }^{24}$ The elastic modulus of the $3 \mathrm{D}$ printed structures was predicted using the generalized form of the Hashin-Shtrikman upper bound for a multiphase composite material. ${ }^{25}$ The Hashin-Shtrikman upper bound is expressed in terms of the elastic modulus of the material constituents using 


$$
\begin{aligned}
E_{\text {calcd }} \leq & {\left[\sum_{i=1}^{N} \phi_{i}\left(\frac{E_{\max }^{*}}{3(1-2 v)}+\frac{E_{i}}{3(1-2 v)}\right)^{-1}\right]^{-1} } \\
& -\frac{E_{\max }^{*}}{3(1-2 v)}
\end{aligned}
$$

Where $E_{\text {calcd }}$ is the calculated upper bound of the bulk modulus for the composite material, $N$ is the total number of phases in the composite, $\phi_{i}$ is the volume fraction of a given phase, $E_{i}$ the elastic modulus of the individual phase materials, $\nu$ is the Poisson's ratio of the given phase measured acoustically, ${ }^{26}$ and $G_{\max }$ is the maximum shear modulus contained within the composite where $E^{*}{ }_{\max } /(1-2 \nu)=4 G_{\max }$. A plot of the calculated elastic modulus for the Hashin-Shtrikman upper bound condition against the measured elastic modulus from ultrasound measurements are shown in Figure 6 below. The

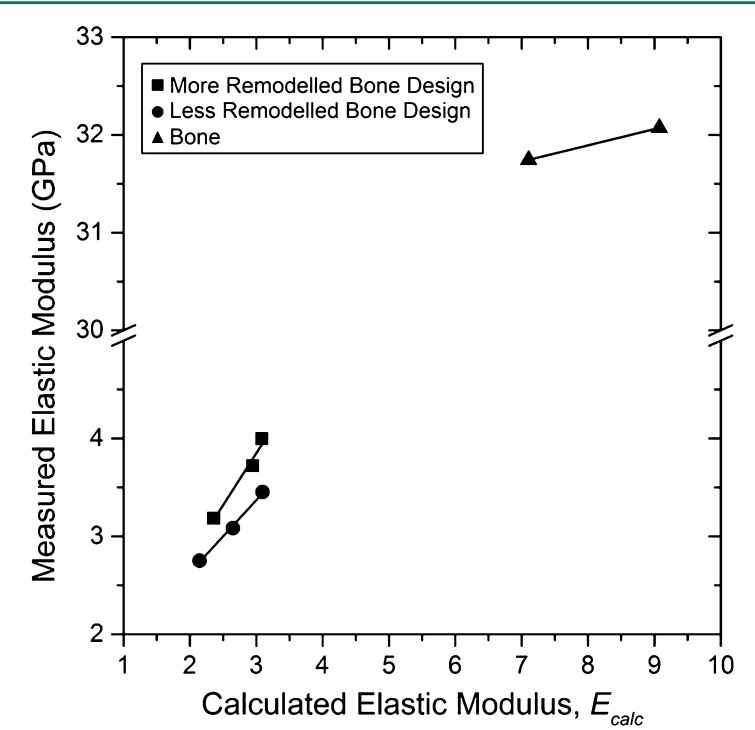

(GPa)

Figure 6. Plot of the measured elastic modulus for bone and 3D printed structures and the corresponding calculated elastic values using the Hashin-Shtrikman upper bound described by eq 4 .

calculated elastic modulus values show somewhat comparable values to the measured elastic modulus values for the $3 \mathrm{D}$ printed structures. Further calculations of the elastic modulus for the bone samples using eq 4 were attempted by incorporating elastic modulus values for the more disordered and ordered collagen structures representative of primary and secondary bone $\mathrm{e}^{5}$ but the resultant correlation with measured elastic modulus values is poor, potentially because of isotropic assumptions in eq 4 applied to more anistropic constituent behavior in bone. The Hashin-Shtrikman model is limited in predicting the bone elastic modulus but more effective in determining the elastic modulus of the $3 \mathrm{D}$ printed composites. The $3 \mathrm{D}$ printed materials are amorphous and isotropic, lacking the anisotropy of the materials found in bone such as collagen. ${ }^{1,2}$ However, potential geometric features could be incorporated into the printed design to replicate anisotropy but is not considered in this current work. Additionally, the Hashin-Shtrikman model assumes interfaces are elastic and the 3D printed materials are also expected to have strong effective interfaces. Such a statement can be partially justified by the calculated elastic modulus fitting more closely to the experimentally measured elastic modulus for the $3 \mathrm{D}$ printed samples. Bone is known to have weak interfaces ${ }^{3}$ and therefore contributes toward a discrepancy between the calculated elastic modulus for the remodelled bone and the experimental measurement. We note that the ultrasonic methods of measuring the elastic modulus of bone tends to give significantly higher results than other mechanical testing techniques. $^{27}$ The trend of increasing elastic modulus as stiffer constituents are used is an obvious outcome from Figure 6. The analytical model of eq 4 is suitable in consistently predicting a higher elastic modulus for the more remodelled bone design across all material compositions compared to the less remodelled bone design. These elastic modulus variations highlights how selecting more appropriate materials, which are currently limited in commercial 3D printing multimaterial systems, will achieve both structural and mechanical fidelity with the imaged tissue.

\section{CONCLUSIONS}

An established workflow that enables the physical output of a 3D printed structure using multiple materials from XCT imaging data has been achieved in this work. Variation in design using less and more remodelled bone samples gave corresponding variability in the elastic modulus of the $3 \mathrm{D}$ printed samples and, combined with a range of mechanically diverse materials, allowed selection of a composite structure with an elastic modulus predicted by an upper bound HashinShtrikman model. The ability to 3D print composite structures from 3D image data sets is a general approach and can be applied to many biological structures provided sufficient imaging contrast is able to discern morphological features and composition, as well as a suitable range of materials providing fidelity with the native tissue considered. Such success will enable improved 3D printed anatomic models and move toward suitable mechano-mimetic structures for potential next-generation patient specific implants.

\section{AUTHOR INFORMATION}

\section{Corresponding Author}

*E-mail: asa.barber@port.ac.uk. ORCID

A. H. Barber: 0000-0003-3334-0266

\section{Notes}

The authors declare no competing financial interest.

\section{ACKNOWLEDGMENTS}

The authors acknowledge Stephan Gehne for his assistance in acoustic measurements, the Future Technology Centre (FTC) at University of Portsmouth for use of facilities, and CDG Ltd. for support in digital facilities.

\section{REFERENCES}

(1) Dunlop, J. W. C.; Fratzl, P. Biological Composites. Annu. Rev. Mater. Res. 2010, 40 (1), 1-24.

(2) Hang, F.; Barber, A. H. Nano-mechanical properties of individual mineralized collagen fibrils from bone tissue. J. R. Soc., Interface 2011, $8,500-505$.

(3) Hang, F.; Gupta, H. S.; Barber, A. H. Nanointerfacial strength between non-collagenous protein and collagen fibrils in antler bone. J. R. Soc., Interface 2014, 11 (92), 20130993.

(4) Oyen, M. L.; Ferguson, V. L.; Bembey, A. K.; Bushby, A. J.; Boyde, A. Composite bounds on the elastic modulus of bone. J. Biomech. 2008, 41 (11), 2585-2588. 
(5) García-Rodríguez, J.; Martínez-Reina, J. Elastic properties of woven bone: effect of mineral content and collagen fibrils orientation. Biomech. Model. Mechanobiol. 2016, 1-14.

(6) Weiner, S.; Wagner, H. D. The Material Bone: StructureMechanical Function Relations. Annu. Rev. Mater. Sci. 1998, 28, 271298.

(7) Madry, H.; van Dijk, C. N.; Mueller-Gerbl, M. The basic science of the subchondral bone. Knee Surgery, Sport. Traumatol. Arthrosc. 2010, 18 (4), 419-433.

(8) Tozzi, G.; De Mori, A.; Oliveira, A.; Roldo, M. Composite hydrogels for bone regeneration. Materials 2016, 9, 267.

(9) Kapadia, B. H.; Berg, R. A.; Daley, J. A.; Fritz, J.; Bhave, A.; Mont, M. A. Periprosthetic joint infection. Lancet 2016, 387, 386-394.

(10) Arabnejad, S.; Burnett Johnston, R.; Pura, J. A.; Singh, B.; Tanzer, M.; Pasini, D. High-strength porous biomaterials for bone replacement: A strategy to assess the interplay between cell morphology, mechanical properties, bone ingrowth and manufacturing constraints. Acta Biomater. 2016, 30, 345-356.

(11) Tozzi, G.; Zhang, Q. H.; Tong, J. 3D real-time micromechanical compressive behaviour of bone-cement interface: Experimental and finite element studies. J. Biomech. 2012, 45 (2), 356-363.

(12) Provaggi, E.; Leong, J. J. H.; Kalaskar, D. M. Applications of 3D printing in the management of severe spinal conditions. Proc. Inst. Mech. Eng., Part H 2016, DOI: 10.1177/0954411916667761.

(13) McGurk, M.; Amis, A. A.; Potamianos, P.; Goodger, N. M. Rapid prototyping techniques for anatomical modelling in medicine. Ann. R. Coll. Surg. Engl. 1997, 79 (3), 169-174.

(14) Malik, H. H.; Darwood, A. R. J.; Shaunak, S.; Kulatilake, P.; ElHilly, A. A.; Mulki, O.; Baskaradas, A. Three-dimensional printing in surgery: a review of current surgical applications. J. Surg. Res. 2015, 199 (2), 512-522.

(15) Farré-Guasch, E.; Wolff, J.; Helder, M. N.; Schulten, E. A. J. M.; Forouzanfar, T.; Klein-Nulend, J. Application of Additive Manufacturing in Oral and Maxillofacial Surgery. J. Oral Maxillofac. Surg. 2015, 73 (12), 2408-2418.

(16) Arora, A.; Datarkar, A. N.; Borle, R. M.; Rai, A.; Adwani, D. G. Custom-made implant for maxillofacial defects using rapid prototype models. J. Oral Maxillofac. Surg. 2013, 71 (2), e104.

(17) Martelli, N.; Serrano, C.; Van Den Brink, H.; Pineau, J.; Prognon, P.; Borget, I.; El Batti, S. Advantages and disadvantages of 3dimensional printing in surgery: A systematic review. Surgery 2016, 159 (6), 1485-1500.

(18) Dimas, L. S.; Bratzel, G. H.; Eylon, I.; Buehler, M. J. Tough composites inspired by mineralized natural materials: Computation, 3D printing, and testing. Adv. Funct. Mater. 2013, 23 (36), 4629-4638.

(19) Tellis, B. C.; Szivek, J. A.; Bliss, C. L.; Margolis, D. S.; Vaidyanathan, R. K.; Calvert, P. Trabecular scaffolds created using micro CT guided fused deposition modeling. Mater. Sci. Eng., C 2008, 28 (1), $171-178$.

(20) Mézière, F.; Juskova, P.; Woittequand, J.; Muller, M.; Bossy, E.; Boistel, R; Malaquin, L.; Derode, A. Experimental observation of ultrasound fast and slow waves through three-dimensional printed trabecular bone phantoms. J. Acoust. Soc. Am. 2016, 139 (2), EL13-18.

(21) Katti, K. S.; Mohanty, B.; Katti, D. R. Nanomechanical properties of nacre. J. Mater. Res. 2006, 21, 1237-1242.

(22) Martínez-Reina, J.; Domínguez, J.; García-Aznar, J. M. Effect of porosity and mineral content on the elastic constants of cortical bone: A multiscale approach. Biomech. Model. Mechanobiol. 2011, 10 (3), 309-322.

(23) International Society for Rock Mechanics Commision on Standardization of Laboratory and Field Tests. Suggested methods for determining sound velocity. Int. J. Rock Mech. Mining Sci. Geomech. Abstr. 1978, 15 (2), 53-58.

(24) Hashin, Z.; Shtrikman, S. A variational approach to the theory of the elastic behaviour of multiphase materials. J. Mech. Phys. Solids 1963, 11 (2), 127-140.

(25) Torquato, S.; Yeong, C. L. Y.; Rintoul, M. D.; Milius, D. L.; Aksay, I. A. Elastic properties and structure of interpenetrating boron carbide/aluminum multiphase composites. J. Am. Ceram. Soc. 1999, 82, $1263-1268$

(26) Kumar, A.; Jayakumar, T.; Raj, B.; Ray, K. K. Correlation between ultrasonic shear wave velocity and Poisson's ratio for isotropic solid materials. Acta Mater. 2003, 51 (8), 2417-2426.

(27) Grimal, Q.; Haupert, S.; Mitton, D.; Vastel, L.; Laugier, P. Assessment of cortical bone elasticity and strength: Mechanical testing and ultrasound provide complementary data. Med. Eng. Phys. 2009, 31 (9), 1140-1147. 